\title{
Papel de los microARN en la patogénesis del cáncer de próstata
}

\author{
The role of microRNAs in the pathogenesis of prostate cancer \\ María José Fernández-Turizo', María Camila Galindo-Quintero', Guillermo Andrés \\ Mosquera-Lizcano', Oriana Echavarría-Cano', Yuliana Marcela Gallo-García CvLAC
}

Fecha correspondencia:

Recibido: junio 4 de 2020.

Revisado: enero 21 de 2021.

Aceptado: febrero 22 de 2021.

Forma de citar:

Fernández Turizo MJ, Galindo Quintero MC, Mosquera Lizcano GA, Echavarría Cano 0, Gallo García YM. Papel de los microARN en la patogénesis del cáncer de próstata. Rev CES Med. 2021;

35(1): 26-36.

Open access

(C) Derecho de autor

Licencia creative commons

Ética de publicaciones

Revisión por pares

Gestión por Open Journal System

DOl: http://dx.doi.org/10.21615/

cesmedicina.35.1.3

ISSN 0120-8705

e-ISSN 2215-9177

Sobre los autores:

1. Estudiante de Medicina.

Universidad CES.

Comparte

F日G. $898 R^{0}$ 田

\section{Resumen}

El cáncer de próstata es una enfermedad prevalente, generadora de gran morbimortalidad y reportada como la quinta causa de muerte a nivel mundial. Según las estimaciones de GLOBOCAN (Global Cancer Observatory por sus siglas en inglés) para el año 2018 se reportaron 1276106 casos nuevos a nivel mundial. Recientemente, surgen los microARN como una posible estrategia futura como biomarcadores, tanto para el diagnóstico como para el tratamiento de la enfermedad. Los microARN son pequeñas moléculas de ARN que cumplen un papel en la regulación de la expresión génica, por lo que la expresión variable de estas moléculas tiene una función importante en la patogénesis del cáncer de próstata. La revisión de la literatura en diferentes bases de datos permitió evidenciar su papel en la patogénesis del cáncer de próstata. Se sugiere que la expresión diferencial de estas moléculas biológicas podría ser de utilidad en la práctica clínica. En Colombia se encuentra en investigación su utilidad en diferentes enfermedades, por lo cual esta revisión de tema podría contribuir a futuras investigaciones.

Palabras clave: MicroARN; Cáncer de próstata; Biomarcadores; Terapia genética.

\begin{abstract}
Prostate cancer is a prevalent disease, with great morbidity and mortality, reported as the fifth leading cause of death worldwide. According to estimates for 2018 by GLOBOCAN (Global Cancer Observatory), 1276106 new cases of prostate cancer were reported worldwide. Identifying methods that allow an early diagnosis and treatment of the disease is necessary. MicroRNA are a possible future strategy as biomarkers for prostate cancer. These are small RNA molecules, in charge of regulating genetic expression. Their differential expression is relevant in the pathogenesis of prostate cancer. Currently, literature suggests the differential expression of these biological molecules could be a tool in prostate cancer, with clinical utility. In Colombia new research related to microRNA and prostate cancer is being conducted, which justifies the pertinence of this literature review and the contribution it can have on future research.
\end{abstract}

Keywords: MicroRNAs; Prostate cancer, Biomarkers, Genetic therapy. 
2. McS, Ph D. Profesor ciencias básicas Facultad de Medicina, Universidad CES.
Se ha planteado que los microARN contribuyen al desarrollo de la neoplasia prostática, involucrándose en la proliferación celular, migración e invasión tisular, angiogénesis, metabolismo tumoral e invasión inmunológica.

\section{Introducción}

El cáncer de próstata es la quinta causa de muerte en el mundo. Según las estimaciones de GLOBOCAN para el año 2018 se reportaron 1276106 casos nuevos a nivel mundial. Respecto a la epidemiología local, el análisis de la situación en salud en Colombia para el 2019 reporta que el cáncer de próstata es el segundo cáncer más común, el tercero en mortalidad con un 7,3 \% y tasa de 15,34 por cada 100000 hombres $(1,2)$.

Diversas moléculas biológicas se han relacionado con la patogénesis del cáncer. Entre estas, se ha planteado que los microARN contribuyen al desarrollo de la neoplasia prostática, involucrándose en la proliferación celular, migración e invasión tisular, angiogénesis, metabolismo tumoral e invasión inmunológica.

Los microARN son pequeñas moléculas endógenas de ARN no codificante, compuestas por 18 a 22 nucleótidos, encargadas de regular la expresión génica.

La biogénesis de estas moléculas se compone de diferentes pasos. Inicialmente, los microARN se transcriben en el núcleo a partir de precursores largos de ARN llamados microARN primarios o (pri-microARN, del inglés primary transcritps) el cual es recortado posteriormente por la enzima Drosha (ribonucleasa ARN-específica doble-trenzada) en el núcleo para producir pre-miRNA con las estructuras específicas de la horquilla de 70 nucleótidos (también llamadas los precursores de la horquilla), este es exportado desde el núcleo al citoplasma por la exportina 5. En el citoplasma, la proteína Dicer y la proteína quinasa activadora dependiente de ARN de cadena doble inducible por interferón forman el complejo de procesamiento de los pre-microARN. Al ser exportados los pre-microARN al citoplasma son reconocidos por este complejo, el cual tiene como finalidad la formación de una cadena madura de microARN y su cadena complementaria, denominada la cadena pasajera. Posteriormente, el complejo se une a la proteína Argonauta 2, lo cual genera el complejo silenciador inducido por microARN (miRISC). Este complejo se encarga de seleccionar la cadena madura encargada de liderar el silenciamiento. El microARN se unirá a su blanco biológico de forma complementaria generando inhibición de la traducción o desestabilización del ARNm blanco (3).

Su papel en la carcinogénesis está vinculado a las diferentes mutaciones que ocurren en las células tumorales; la expresión desregulada de los microARN, ocurre a través de varios mecanismos, tales como amplificación o deleción de genes, cambios epigenéticos, regulación de los factores de transcripción o modificación postranscripcional. La expresión alterada, repercute en las características del cáncer, el mantenimiento de la señalización proliferativa, evasión de los supresores de crecimiento, resistencia a la muerte celular, activación de la invasión y metástasis, así como la inducción de angiogénesis. La identificación de los patrones de aumento o disminución de los microARN, podría tener utilidad para establecer el diagnóstico temprano, tratamiento y pronostico en el cáncer de próstata (4-8).

Esta revisión tiene como objetivo presentar y sintetizar la evidencia científica disponible a la fecha sobre la relación de los microARN y la patogénesis del cáncer de próstata. 


\section{Métodos}

Se realizó una recolección de artículos en los idiomas inglés y español en PubMed, ScienceDirect, Medline complete, Cochrane, Scopus y UpToDate. Se seleccionaron publicaciones de los últimos seis años y se complementó la búsqueda mediante la selección de bibliografía referenciada en los textos identificados inicialmente, además de información encontrada en artículos con fecha anterior de publicación.

\section{Generalidades}

Los microARN son pequeñas secuencias de ARN no codificante, sintetizadas en el núcleo. Se encargan de regular el papel de los ARNm y su expresión génica al inhibir el proceso de traducción. La proteína resultante de la traducción del ARNm en específico se disminuye, por lo cual se ven afectados todos los procesos biológicos en los

Los microARN son pequeñas secuencias de ARN no codificante, sintetizadas en el núcleo. Se encargan de regular el papel de los ARNm y su expresión génica al inhibir el proceso de traducción. En el caso de las neoplasias, los microARN estimulan oncogenes -conocidos como oncomiRs-, o alteran el funcionamiento normal de los supresores tumorales. que participa. Específicamente, en el caso de las neoplasias, los microARN estimulan oncogenes -conocidos como oncomiRs-, o alteran el funcionamiento normal de los supresores tumorales. Los microARN se expresan de forma diferencial en las diferentes etapas del desarrollo de la neoplasia prostática $(9,10)$.

\section{Proliferación y crecimiento celular tumoral}

La activación anormal de la vía del PI3K (phosphatidylinositol 3-kinase por sus siglas en inglés) (figura 1) resulta en la alteración de los mecanismos que controlan el crecimiento celular, lo cual favorece la proliferación descontrolada. La vía de PI3K es fundamental en las diferentes neoplasias, entre estas el cáncer de próstata (11). Específicamente, miR-129, miR45-30, miR-224-5p, miR-16-5p y el miR-486-5p se han asociado a la supresión de la vía del PI3K al inhibir factores de transcripción miembros de la familia SMAD (segundo mensajero encargado de llevar información al núcleo celular) (12).

El miR-30e, a su vez, inhibe la activación de las MAPK (Mitogen-Activated Protein Kinases por sus siglas en inglés), impactando igualmente en la supervivencia tumoral. El miR-1 inhibe la proliferación celular al bloquear la señalización mediante el blanco AKT (proteína cinasa B, por sus siglas en inglés). Por el contrario, se ha reportado que la sobreexpresión de miR-410-3p promueve la proliferación de células tumorales prostáticas al estimular la señalización por AKT y disminuir la señalización por PTEN (phosphatidylinositol-3,4,5-trisphosphate 3-phosphatase, por sus siglas en inglés) (13-17) (cuadro). 
En lo referente a microARN en el metabolismo celular en el cáncer de próstata, se ha reportado la interacción principalmente de miR-205, miR142 y miR-181b, estimulando la captación y la utilización de glucosa. La disminución en la expresión del miR-132 se asocia con una regulación al alta de GLUT1 (glucose transporter 1, por sus siglas en inglés), lo que ocasiona una mayor captación de glucosa y secreción de lactato, favoreciendo el crecimiento tumoral.

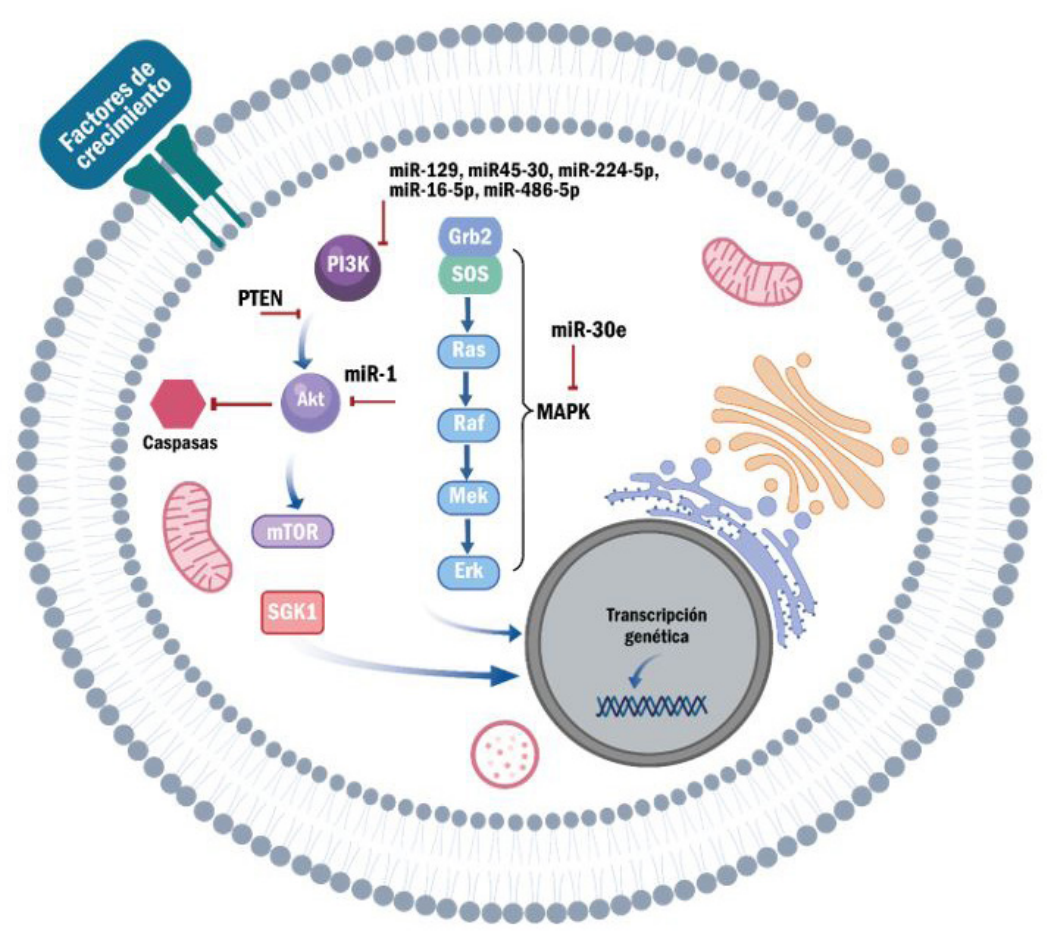

Figura 1. Vía de la PI3K y MAPK

Akt: Proteína serina-treonina cinasa, Grb2: Proteína adaptadora, MAPK: Cinasas activadas por mitógenos, mTOR: Blanco de rapamicina en mamíferos, PI3K: Fosfatidilinositol- 3-cinasa, PTEN: Fosfatidilinositol-3,4,5-trifosfato 3-fosfatasa, SGK1: Cinasa 1sérica regulada por glucocorticoides, SOS: Proteína adaptadora. Ras, Raf, Mek, Erk: Proteínas cinasas.

Fuente: Elaboración propia.

\section{Migración tumoral e invasión tisular}

La E-cadherina evita la invasión tisular y disminuye la migración celular. Específicamente, la cadherina 11 se expresa de forma aberrante en las células cancerígenas prostáticas, facilitando la invasión del hueso por estas células (18-20). La familia miR200 se une a ZEB1 y ZEB2 (zinc finger E-box-binding homeobox 1 por sus siglas en inglés). Estas son moléculas que actúan como represores de la E-cadherina durante su transcripción, favoreciendo la invasión tumoral. En cuanto al microambiente tumoral se ha visto que la expresión de miR-29b, miR-146a, miR-130b y miR-205 (cuadro) pueden inhibir la actividad proteolítica de la MMP-2 (metaloproteinasa 2) disminuyendo la capacidad de la neoplasia para infiltrar tejidos adyacentes (21).

\section{Inducción de la angiogénesis}

La sobreexpresión de miR-21 en líneas celulares de cáncer de próstata induce angiogénesis, al estimular la expresión de HIF-1 (hypoxia inducible por sus siglas en inglés) y el VEGF (vascular endothelial growth factor, por sus siglas en inglés) (22).

\section{Metabolismo celular en células tumorales}

En lo referente a la participación de los microARN en el metabolismo celular en el cáncer de próstata, se ha reportado la interacción principalmente miR-205, miR-142 y miR-181b estimulando la captación y la utilización de glucosa. La disminución en la expresión del miR-132 se asocia con una regulación al alta de GLUT1 (glucose transporter 1, por sus siglas en inglés), lo que ocasiona una mayor captación de glucosa y secreción de lactato, favoreciendo el crecimiento tumoral (23). 


\section{Evasión inmunológica}

La sobreexpresión de moléculas co-inhibitorias de la activación de células T como B7X, PD-L1 y B7-H3 en células tumorales prostáticas se asocia a una evolución tórpida de la enfermedad. El miR-708-5p controla la proliferación celular al bloquear directamente la molécula co-inhibitoria B7/H3. Por lo anterior, impide la evasión del sistema inmune y permite la activación de las células T (24).

El complejo PD-L1/PD1 es de vital importancia en el comportamiento y la evasión inmune de las células tumorales en el cáncer de próstata. Al unirse PD-L1 (programmed death-ligand 1, por sus siglas en inglés) con PD1 (programmed cell death protein 1 por sus siglas en inglés), se evita que las células T eliminen las células tumorales. Los microARN que se han asociado a la disminución en la expresión de PDL1, son miR-195/-16 y las familias de miR-34 y miR-200 (19,25). En consecuencia, al disminuirse la expresión de PDL1, las células T reconocen las células tumorales (figura 2).

El complejo PD-L1/PD1 es de vital importancia en el comportamiento y la evasión inmune de las células tumorales en el cáncer de próstata.

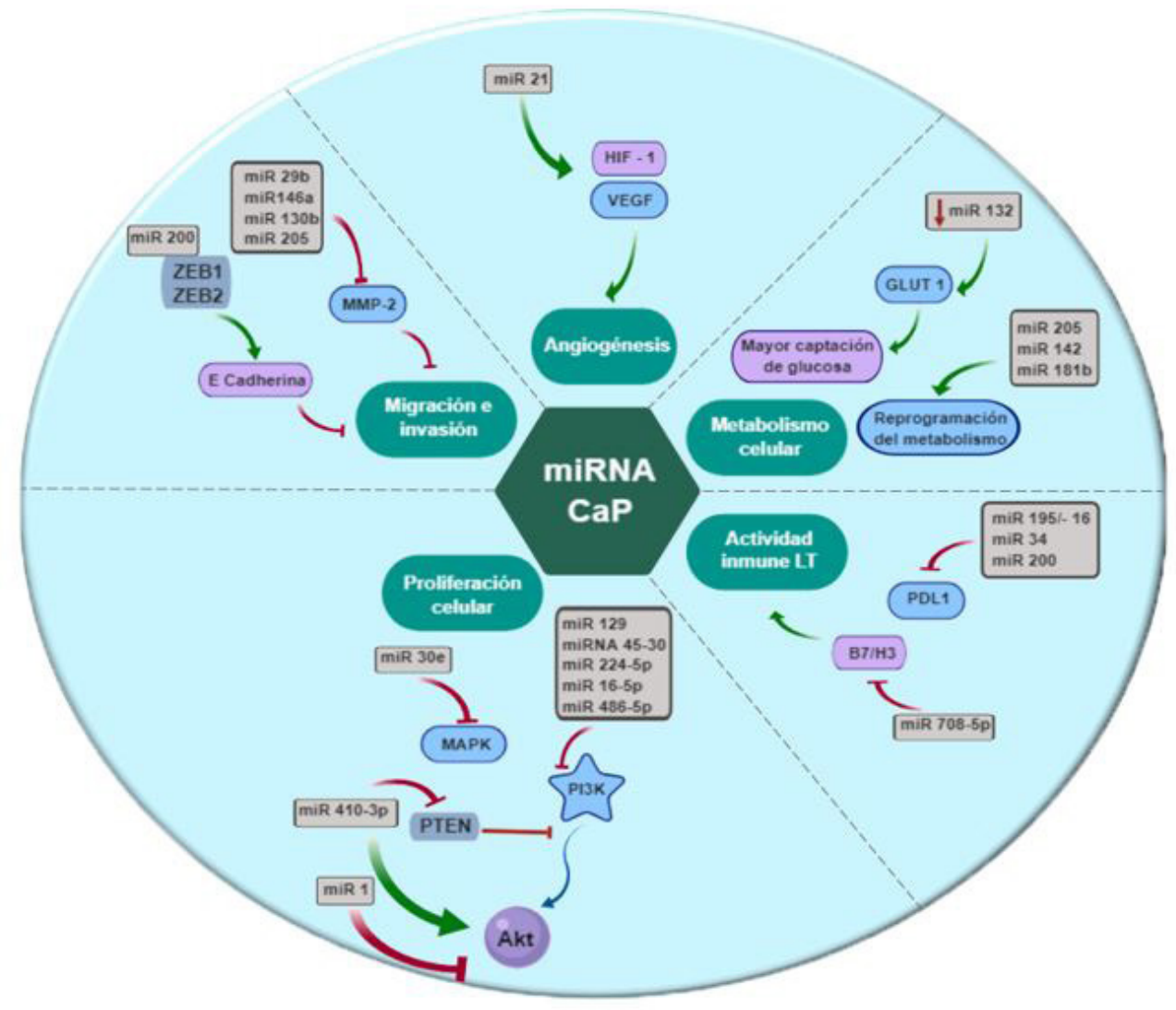

Figura 2. Síntesis de los principales microARN involucrados en la patogénesis del cáncer de próstata.

La señalización verde indica estimulación, señalización roja indica bloqueo de la molécula o el proceso

Akt: Proteína serina-treonina cinasa, CaP: Cáncer de próstata, GLUT 1: Transportador de glucosa 1. HIF 1: Factor inducible por hipoxia 1. LT: Linfocitos T, MAPK: Cinasas activadas por mitógenos, MMP-2: Metaloproteinasa 2, PDL1: Ligando 1 de muerte programada, PI3K: Fosfatidilinositol- 3-cinasa, PTEN: Fosfatidilinositol-3,4,5-trifosfato 3-fosfatasa, VEGF: Factor de crecimiento endotelial vascular, ZEB1:Zinc finger E-Box-binding homeobox 1, ZEB2: Zinc finger E-Box-binding homeobox 2. 
Enero - abril de 2021 - Pág 31

La intervención terapéutica utilizando la transfección de microARN específicos se ha estudiado recientemente. Según el mecanismo de acción conocido de los microARN, la terapia debe ser establecida mediante dos estrategias básicas. Una de ellas es reemplazar los microARN disminuidos por microARN sintéticos, la otra es añadir nucleótidos modificados generando mutaciones denominadas sin sentido, lo que se conoce como (anti-microARN).

\section{MicroARN como biomarcadores prometedores en el cáncer de próstata}

Los microARN se han medido en diferentes tejidos y líquidos corporales. En relación con el cáncer de próstata se ha identificado una sobreexpresión plasmática de miR-21, miR-125b, miR-126, miR-141, let -7, miR-205 y miR-375 (cuadro). De igual forma, en tejido prostático se ha encontrado sobreexpresión de miR-20a y en orina se evidencia un incremento de miR-21, miR-141 y miR-375 (26-28). La identificación de la sobreexpresión de estos microARN podría representar utilidad para el diagnóstico de la enfermedad (3,29-31).

Se sugiere que los microARN podrían tener relevancia al diferenciar las etapas de la enfermedad, entre ellas cáncer localizado o metastásico. Una sobreexpresión de miR-141, miR-200 y miR-375 ha sido reportada en cáncer de próstata avanzado. De igual forma, se han relacionado con la agresividad de la neoplasia prostática miR-17 y miR-192 (32,33).

Respecto a los microARN como biomarcadores en el tratamiento del cáncer de próstata se propone su capacidad de diferenciar entre cáncer de próstata sensible a la castración y resistente a esta. En este último, se encuentran disminuidos los miR-125b-2 y miR-708 y sobreexpresados el miR-375, miR-141, miR-143, miR-362-5p, miR-214, let-7i y miR-545 $(27,29)$.

Se ha corroborado la utilidad de los microARN como moléculas tanto sensibilizantes, como asociadas al desarrollo de resistencia al tratamiento en cáncer de próstata. Se ha identificado la sobreexpresión de miR-34a, miR-205 y miR-31 asociada a la sensibilidad a los taxanos. De igual forma, se asocia la sobreexpresión de miR-106b con la resistencia a la radioterapia. Por el contrario la sobreexpresión de miR449a confiere mayor sensibilidad a esta (34-40).

\section{Aplicabilidad clínica de los microARN}

La importancia clínica del estudio de los microARN radica, no solo en la posibilidad del diagnóstico en etapas tempranas de la enfermedad, lo cual, lleva a un mejor pronostico; si no también el uso de estas moléculas para tratar la neoplasia.

La intervención terapéutica utilizando la transfección de microARN específicos se ha estudiado recientemente y el interés por conocer su utilidad es cada vez mayor. Según el mecanismo de acción conocido de los microARN, la terapia debe ser establecida mediante dos estrategias básicas. Una de ellas es reemplazar los microARN disminuidos por microARN sintéticos, la otra es añadir nucleótidos modificados generando mutaciones denominadas sin sentido, lo que se conoce como (anti-microARN). Actualmente se encuentran en desarrollo las terapias para el CaP utilizando microARN. Deben surgir más estudios que evalúen la respuesta a la terapia génica en humanos, ya que hasta ahora los anteriores se unen con colesterol, dextrano, fosfato de calcio, liposomas, dendrímeros, nanopartículas, entre otros compuestos, e ingresan a la célula mediante endocitosis. En relación a los mecanismos físicos, se puede transferir el microARN por medio de electroporación o microinyección $(41,42)$.

Respecto a la transfección, se ha visto que el mecanismo más eficaz de entrega de los microARN es mediante el uso de nanopartículas. Un estudio realizado demostró la efectividad experimental de este mecanismo, para la transfección in vitro del miR-145 a las células tumorales de próstata (43). 
Se evidencia la identificación mediante el uso de la técnica RT-qPCR de los microARN miR-129, miR-30e, miR-1, miR-410-3p, miR-205, miR21 y miR-20a como potenciales biomarcadores del cáncer de prostata en estadio temprano.
En la actualidad, se puede evidenciar cómo el conocimiento de los microARN, ha permitido el estudio de diferentes drogas experimentales que tienen como diana terapéutica estas biomoléculas, entre estos Miravirsen, el cual es un antimicroARN del miR-122, utilizado en el tratamiento de la hepatitis C, el cual se encuentra en estudios clínicos fase 2. Respecto al uso clínico de estas moléculas en cáncer, el MRX34, la cual es una reconstrucción sintética del miR-34a (44-46).

Cuadro. Principales microARN reportados como potenciales biomarcadores

\begin{tabular}{|c|c|c|c|}
\hline microARN & Expresión con -Utilidad clínica & Técnica detección & Estudio \\
\hline miR-129 & Aumentado - Inhibe PI3K & \multirow{8}{*}{$R T-q P C R$} & Yunpeng Jia Et al (12). \\
\hline miR-30e & Aumentado - Inhibe MAKP & & Xin-Min Zheng Et al (15). \\
\hline miR-1 & Aumentado - Inhibición AKT & & Sing Gao Et al (16). \\
\hline $\operatorname{miR}-410-3 p$ & Disminuido - Inhibe AKT y PTEN & & Yuelong Zhang Et al (17). \\
\hline miR-205 & Aumentado - Inhibe la MMP-2 & & Ning Wang Et al (21). \\
\hline miR-21 & Disminución - Inhibe HIF-1ª & & Ling-Zhi Liu Et al (22). \\
\hline miR-20a & $\begin{array}{c}\text { Aumentado - Detección de células } \\
\text { tumorales no diferenciadas }\end{array}$ & & M.Pesta Et al (26). \\
\hline miR-375 & $\begin{array}{c}\text { Aumentado - Predicción de riesgo } \\
\text { de metástasis }\end{array}$ & & David Bidarra Et al (27). \\
\hline
\end{tabular}

RT-qPCR: Quantitative reverse transcription polymerase por sus siglas en ingles.

\section{Conclusiones}

Durante la revisión de los diferentes estudios se evidencia de una manera sólida la identificación mediante el uso de la técnica RT-qPCR de los microARN miR-129, miR-30e, miR-1, miR-410-3p, miR-205, miR-21 y miR-20a como potenciales biomarcadores del cáncer de próstata en estadio temprano.

La expresión diferencial de los microARN en el cáncer de próstata es un tema que aún continua en estudio. Colombia, aún no cuenta con estudios experimentales publicados y enfocados a la medición de la expresión diferencial de los microARN en población local con cáncer de próstata. Sin embargo, se encuentran disponibles revisiones de tema, en relación con la utilidad de estas moléculas en diferentes escenarios clínicos.

Las revisiones publicadas sugieren la relación de los microARN con la patogénesis del cáncer de próstata y su pertinencia como biomarcadores para el diagnóstico y tratamiento de la enfermedad. Evidenciando la necesidad de que la comunidad científica de Colombia inicié investigaciones en esta área en la población local con miras a su uso en la práctica clínica.

\section{Conflicto de intereses}

Ninguno de los autores reporta un potencial conflicto de intereses en la realización del artículo.

\section{Financiación}

El estudio no contó con financiación externa, por lo que esta no influenció su realización. 


\section{Bibliografía}

1. Rawla P. Epidemiology of prostate cancer. World J Oncol. 2019; 10(2):63-89.

2. Ministerio de Salud y Protección Social. Análisis de Situación de Salud (ASIS) Colombia, [en línea] 2020. [citada 2020 junio 2]. Hallado URL: https://www.minsalud.gov.co/sites/rid/Lists/BibliotecaDigital/RIDE/VS/ED/PSP/asis-2019-co$\underline{\text { lombia.pdf }}$

3. Jackson BL, Grabowska A, Ratan H.L. MicroRNA in prostate cancer: functional importance and potential as circulating biomarkers. BMC Cancer. 2014; 14:930.

4. Movahedpour A, Ahmadi N, Ghasemi Y, Savardashtaki A, Shabaninejad Z. Circulating microRNAs as potential diagnostic biomarkers and therapeutic targets in prostate cancer: Current status and future perspectives. J Cell Biochem. 2019; 120 (10): 16316-16329.

5. Santos PB, Patel H, Henrique R, Félix A. Can epigenetic and inflammatory biomarkers identify clinically aggressive prostate cancer? World J Clin Oncol. 2020; 11(2): 43-52.

6. Jin W, Fei $X$, Wang $X$, Song $Y$, Chen F. Detection and Prognosis of prostate cancer using blood-based biomarkers. Mediators Inflamm 2020; 2020: 8730608.

7. Khan S, Ayub H, Khan T, Wahid F. MicroRNA biogenesis, gene silencing mechanisms and role in breast, ovarian and prostate cancer. Biochimie. 2019; 167:12

8. Wei J, Yin Y, Deng Q, Zhou J, Wang Y, Yin G, et al. Integrative analysis of MicroRNA and gene interactions for revealing candidate signatures in prostate cancer. Front Genet. 2020; 11: 176.

9. Matin F, Jeet V, Clements JA, Yousef GM, Batra J. MicroRNA theranostics in prostate cancer precision medicine. Clin Chem. 2016; 62(10):1318-1333.

10. Yan JW, Lin JS, He XX. The emerging role of miR-375 in cancer. Int. J. Cancer. 2014; 135: 1011-1018

11. Pinzón CE, Serrano ML, Sanabria MC. Papel de la vía fosfatidilinositol 3 kinasa (PI3K/Akt) en humanos. Revista Ciencias de la Salud. 2009. 7(2).

12. Jia Y, Gao Y, Dou J. Effects of miR-129-3p on biological functions of prostate cancer cells through targeted regulation of Smad3. Oncol Lett. 2020; 19(2):1195-1202.

13. Shankar E, Weis MC, Avva J, Shukla S, Shukla M, Sreenath SN, et al. Complex Systems biology approach in connecting PI3K-Akt and NF-KB pathways in prostate cancer. Cells. 2019; 8(3):201.

14. Yang J, Nie J, Ma X, Wei Y, Peng Y, Wei X. Targeting PI3K in cancer: Mechanisms and advances in clinical trials. Molecular Cancer. 2019; 26 (18). 
15. Zheng XM, Zhang P, Liu MH, Chen P, Zhang WB. MicroRNA-30e inhibits adhesion, migration, invasion and cell cycle progression of prostate cancer cells via inhibition of the activation of the MAPK signaling pathway by downregulating CHRM3. Int J Oncol. 2019; 54(2):443-454.

16. Gao S, Zhao Z, Wu R, Wu L, Tian X, Zhang Z. MiR-1 inhibits prostate cancer PC3 cells proliferation through the Akt/mTOR signaling pathway by binding to c-Met. Biomed Pharmacother. 2019; 109:1406-1410.

17. Zhang Y, Zhang D, Lv J, Wang S, Zhang Q. miR-410-3p promotes prostate cancer progression via regulating PTEN/AKT/mTOR signaling pathway. Biochem Biophys Res Commun. 2018; 503(4):2459-2465.

18. Tripathi V, Popescu N, Zimonjic, D. DLC1 induces expression of E-cadherin in prostate cancer cells through Rho pathway and suppresses invasion. Oncogene. $2014 ; 33: 724-733$.

19. Tao Z, Xu S, Ruan H, Wang T, Song W, Qian L, Chen K: MiR-195/-16 Family Enhances Radiotherapy via T Cell Activation in the Tumor Microenvironment by Blocking the PD-L1 Immune Checkpoint. Cell Physiol Biochem. 2018; 48:801-814.

20. Huang CF, Lira C, Chu K, et al. Cadherin-11 increases migration and invasion of prostate cancer cells and enhances their interaction with osteoblasts. Cancer Res. 2010; 70(11):4580-4589.

21. Wang N, Li Q, Feng NH, et al. miR-205 is frequently downregulated in prostate cancer and acts as a tumor suppressor by inhibiting tumor growth. Asian $\mathrm{J}$ Androl. 2013; 15(6):735-741.

22. Liu LZ, Li C, Chen Q, Jing Y, Carpenter R, Jiang Y, et al. MiR-21 Induced Angiogenesis through AKT and ERK Activation and HIF-1? Expression. PLOS ONE. 2011; 6(4): e19139.

23. Kasomva K, Sen A, Paulraj MG, Sailo S, Raphael V, Puro K u, et al. Roles of microRNA in prostate cancer cell metabolism. Int J Biochem Cell Biol. 2018; 102:109-116.

24. Barach YS, Lee JS, Zang X. T cell coinhibition in prostate cancer: new immune evasion pathways and emerging therapeutics. Trends Mol Med. 2011; 17(1):47-55.

25. Cortez MA, Ivan C, Valdecanas D, Wang X, Peltier HJ, Ye Y, et al. PDL1 Regulation by p53 via miR-34. J Natl Cancer Inst. 2015; 108(1):djv303.

26. Pesta M, Klecka J, Kulda V, Topolcan O, Hora M, Eret M, et al. Importance of miR-20a expression in prostate cancer tissue. Anticancer Res. 2010; 30(9):3579-3583.

27. Bidarra D, Constâncio V, Barros-Silva D, Ramalho-Carvalho J, Moreira-Barbosa C. Antunes A, et al. Circulating MicroRNAs as Biomarkers for Prostate Cancer Detection and Metastasis Development Prediction. Front Oncol. 2019; 9:900.

28. Vanacore D, Boccellino M, Rossetti S, Cavaliere C, D'Aniello C, Di Franco R, et al. Micrornas in prostate cancer: an overview. Oncotarget. 2017; 8(30):50240-50251. 
29. Guo X, Han T, Hu P, Guo X, Zhu C, Wang Y, et al. Five microRNAs in serum as potential biomarkers for prostate cancer risk assessment and therapeutic intervention. Int Urol Nephrol. 2018; 50(12):2193-2200.

30. Lyu J, Zhao L, Wang F, Ji J, Cao Z, Xu H, et al. Discovery and validation of serum MicroRNAs as early diagnostic biomarkers for prostate cancer in Chinese population. Biomed Res Int. 2019.

31. Jin W, Fei X, Wang $X$, Chen F, Song Y. Circulating miRNAs as biomarkers for prostate cancer diagnosis in subjects with benign prostatic hyperplasia. J Immunol Res. 2020; 2020:5873056.

32. Farran B, Dyson G, Craig D, Dombkoski A, Beebe-Dimmer JL, Powell IJ, et al. A study of circulating microRNAs identifies a new potential biomarker panel to distinguish aggressive prostate cancer. Carcinogenesis. 2018; 39(4):556-561.

33. Shukla KK, Misra S, Pareek P, Mishra V, Singhal B, Sharma P, et al. Recent scenario of microRNA as diagnostic and prognostic biomarkers of prostate cancer. Urologic Oncology. 2017; 35(3):92-101.

34. Wang Y, Lieberman R, Pan J, Zhang Q, Du M, Zhang P, et al. miR-375 induces docetaxel resistance in prostate cancer by targeting SEC23A and YAP1. Mol Cancer. 2016; 15(1):70.

35. Mao A, Zhao Q, Zhou X, Sun C, Si J, Zhou R, et al. MicroRNA-449a enhances radiosensitivity by downregulation of c-Myc in prostate cancer cells. Sci Rep. 2016; 6:27346.

36. Thieu W, Tilki D, de Vere White R, Evans CP. The role of microRNA in castration-resistant prostate cancer. Urol Oncol. 2014; 32(5):517-523.

37. Bhatnagar N, Li X, Padi SK, Zhang Q, Tang MS, Guo B. Downregulation of miR-205 and miR-31 confers resistance to chemotherapy-induced apoptosis in prostate cancer cells. Cell Death Dis. 2010; 1(12):e105.

38. Nagesh PKB, Chowdhury P, Hatami E, Boya VKN, Kashyap VK, Khan S, et al. miRNA-205 nanoformulation sensitizes prostate cancer cells to chemotherapy. Cancers (Basel). 2018; 10(9):289.

39. Ni J, Bucci J, Chang L, Malouf D, Graham P, Li Y. Targeting MicroRNAs in Prostate Cancer Radiotherapy. Theranostics. 2017; 7(13):3243-3259.

40. Li B, Shi XB, Nori D, et al. Down-regulation of microRNA $106 \mathrm{~b}$ is involved in p21-mediated cell cycle arrest in response to radiation in prostate cancer cells. Prostate. 2011; 71(6):567-574.

41. Shah MY, Ferrajoli A, Sood AK, Lopez-Berestein G, Calin GA. microRNA therapeutics in cancer - an emerging concept. EBioMedicine. 2016;12:34-42.

42. Kim TK, Eberwine JH. Mammalian cell transfection: the present and the future. Anal Bioanal Chem. 2010;397(8):3173-3178. 
43. Ekin A, Karatas OF, Culha M, Ozen M. Designing a gold nanoparticle-based nanocarrier for microRNA transfection into the prostate and breast cancer cells. J Gene Med. 2014, 16(11-12):331-5.

44. Bonneau E, Neveu B, Kostantin E, Tsongalis GJ, De Guire V. How close are miRNAs from clinical practice? A perspective on the diagnostic and therapeutic market. EJIFCC. 2019;30(2):114-127.

45. Hong, D.S., Kang, YK., Borad, M. et al. Phase 1 study of MRX34, a liposomal miR-34a mimic, in patients with advanced solid tumours. Br J Cáncer. 2020, $122,1630-1637$.

46. Chakraborty C, Sharma AR, Sharma G, Lee SS. Therapeutic advances of miRNAs: A preclinical and clinical update. J Adv Res. 2020; 28:127-138. 